\title{
Evaluación de Tecnologías en Salud: Un Enfoque Hospitalario para la Incorporación de Dispositivos Médico
}

\section{Assessment of Health Technologies: A Hospital Approach for the Incorporation of Medical Devices}

\section{P. A. Lizcano-Jaramillo, J. E. Camacho-Cogollo}

Universidad EIA

\section{RESUMEN}

Este artículo presenta el estado del arte acerca de las evaluaciones de tecnología y los aportes que han sido realizados a nivel hospitalario en diversos países del mundo. La incorporación de tecnologías sanitarias está enfocado al mejoramiento de la calidad y la eficiencia en la prestación de los servicios de salud. La evaluación de tecnología son un proceso científico, multidisciplinario y multidimensional que tiene como objetivo contribuir y mejorar la toma de decisiones en los procedimientos de adquisición e incorporación tecnológica, mediante la evaluación comparativa de los efectos positivos y negativos de las tecnologías en hospitales. El propósito generalmente considera diversas alternativas, proporcionando la mejor selección de tecnología que compense las necesidades médicas en instituciones de salud. A nivel mundial han trabajado constantemente para mejorar los procesos de evaluación de tecnología en el ámbito nacional y hospitalario. La implementación de las metodologías de evaluación en hospitales permite contribuir a la disminución de los riesgos generados por el uso de los dispositivos o equipos médicos en relación con la seguridad de los pacientes implementando tecnologías eficaces para diversos tratamientos. En conclusión, la evaluaciones de tecnología es un proceso fundamental que proporciona información relevante para la toma de decisiones para las incorporaciones de nuevos dispositivos médicos en hospitales. 
This article presents the state of the art about the evaluations of technology and the contributions that have been made at a hospital level in different countries of the world. The incorporation of health technologies is focused on the improvement of quality and efficiency in the provision of health services. The evaluation of technology is a scientific, multidisciplinary and multidimensional process that aims to contribute and improve decision making in the procedures of acquisition and technological incorporation, through the comparative evaluation of the positive and negative effects of technologies in hospitals. The purpose generally considers various alternatives, providing the best selection of technology that compensates for medical needs in health institutions. At the global level, they have constantly worked to improve the technology assessment processes at the national and hospital levels. The implementation of evaluation methodologies in hospitals allows contributing to the reduction of the risks generated using medical devices or equipment in relation to patient safety by implementing effective technologies for various treatments. In conclusion, the technology assessment is a fundamental process that provides relevant information for decision making for the incorporation of new medical devices in hospitals.

KEYWORDS: incorporation of technology; health technology evaluation; decision making; evaluation criteria

\section{Correspondencia}

DESTINATARIO: Paula Andrea Lizcano Jaramillo

INSTITUCIÓN: Universidad EIA

DIRECCIÓN: Calle 25 Sur \#42-73, C. P. 055420,

Envigado, Colombia

CORREO ELECTRÓNICO: paula.lizcano@eia.edu.co

\section{Fecha de recepción:}

15 de junio de 2019

Fecha de aceptación:

15 de agosto de 2019 


\section{INTRODUCCIÓN}

La incorporación de tecnologías sanitarias es un proceso enfocado al mejoramiento de la calidad y eficiencia en la prestación de los servicios de salud a través de la implementación de tecnologías efectivas. Dentro de los procesos de incorporación se destacan puntos importantes como la evaluación técnica y clínica con el objetivo de recolectar, analizar, sintetizar información y conocimiento para contribuir a la mejora de toma de decisiones para adquirir nuevas tecnologías en la práctica médica y en las políticas de salud ${ }^{[1]}$. Cuando se habla de incorporación tecnológica se hace referencia al proceso de evaluación de tecnología en salud (ETS). De acuerdo con la Organización Mundial de la Salud (OMS) existen organizaciones especializadas en la evaluación de tecnologías sanitarias que se están convirtiendo en elementos institucionales de los sistemas de salud, no solo para ayudar a identificar tecnologías sanitarias poco seguras e ineficaces, sino también para detectar tecnologías prometedoras que puedan estimular la innovación de dispositivos y equipos médicos en los países y en las instituciones prestadoras de los servicios de salud ${ }^{[2]}$. A nivel internacional se han creado organismos como la Sociedad Internacional de Evaluación de Tecnologías Sanitarias (HTAi) y la Red Internacional de Agencias de Evaluación de Tecnologías Sanitarias (INAHTA), en compañía de la OMS, se han comprometido fuertemente con el desarrollo de las ETS ${ }^{[2]}$. Actualmente, se reconoce que los productos de la ETS son procedimientos pertinentes para la toma de decisiones a nivel gubernamental o institucional y se utiliza eficazmente en diversos países del mundo [3]. En países de ingresos medios como: Argentina, Brasil, México, entre otros, se ha estudiado el desarrollo y la institucionalización de las ETS ${ }^{[4]}$. Para lograr este objetivo, se han dedicado fundamentalmente a instruir y capacitar al personal nuevo para la realización de ETS. Sin embargo, esto no es suficiente y es necesario considerar aspectos como: la realización de actividades de identificación, priorización y evaluación de nuevas tecnologías, informar, divulgar e implementar las evaluaciones desarrolladas ${ }^{[5]}$. El enfoque que han tenido las ETS es en el análisis de aspectos clínicos y económicos. Por lo general, las evaluaciones de tecnología han orientado la mayoría de sus desarrollos en el área farmacológica, debido que estos productos son una de las principales áreas de investigación más avanzadas. Aunque varios países cuentan con institutos de ETS que incluyen tecnologías como, los dispositivos y equipos médicos, los informes producidos para tecnologías no farmacológicas sigue siendo bajo ${ }^{[6]}$. Para el año 2011, en Colombia se da la conformación del Instituto de Evaluación de Tecnologías en Salud (IETS) organización establecida por el Ministerio de Salud colombiano y el Invima, la cual se encarga de desarrollar ETS con base en evidencia científica a nivel gubernamental. Si bien este Instituto realiza sus informes basados en criterios de evaluación como seguridad, eficacia y económico, el enfoque que se ha dado no da cobertura a otras necesidades de evaluación de tecnologías en el ámbito hospitalario ${ }^{[7]}$. Las ETS brindan soporte en el momento de la toma de decisiones, en ocasiones este se dificulta por diversos factores, como por ejemplo los dispositivos y equipos médicos que son nuevos en el mercado y que en ocasiones son de cuestionable eficacia; además, su incorporación progresiva en instituciones de salud, el costo y como controlarlo, son unas de las variables predominantes. Muchas de estas incorporaciones se realizan antes de que puedan evaluarse con rigurosidad los impactos clínicos, económicos, sociales y éticos. En algunos casos, son incompatibles con la infraestructura hospitalaria, generando gastos de mantenimiento adicionales e incidentes por mal uso debido a la falta de entrenamiento y el poco impacto funcional entre los usuarios. Seguidamente la información hallada en el medio para evaluar las diversas tecnologías es imprecisa e insuficiente lo que dificulta el proceso de decisión. Todos estos factores conllevan a la realización de inversiones de mediana o alta cuantía que no satisfacen las necesidades hospitalarias ${ }^{[8]}$. 


\section{Gestión tecnológica}

En un sistema de salud las tecnologías sanitarias son fundamentales para aquellos que se encuentran prestando servicios de salud. Los dispositivos médicos son cruciales para la prevención, diagnóstico, tratamiento y rehabilitación de enfermedades, respecto a estos dispositivos se han tratado temas derivados con la instalación y el uso inadecuado, así como la necesidad de establecer prioridades en la selección y gestión de tecnologías sanitarias, en especial los dispositivos médi$\cos ^{[9]}$. La gestión tecnológica contribuye a la adopción y ejecución de las decisiones sobre políticas, estrategias, planes y acciones relacionadas con la creación, difusión y uso de la tecnología ${ }^{[10]}$. Lo anterior permite abrir un panorama que incluye procedimientos de planificación, evaluación de necesidades, selección, adquisición, donaciones, inventarios, instalación, mantenimiento de equipos médicos, capacitación para el uso seguro y disposición final de la tecnología, entre otras actividades. Una gestión tecnológica en salud bien implementada ofrece óptimos resultados, a través de una buena implementación de los recursos ${ }^{[11]}$.

\section{Evaluación de tecnología}

Las ETS es uno de los procesos asociados a la gestión tecnológica en salud, la cual se define como un proceso científico, multidisciplinario, y multidimensional para la toma de decisiones, que permite realizar una evaluación comparativa de los efectos positivos y negativos de las tecnologías, cuyo propósito es generalmente considerar diversas alternativas, proporcionando la mejor selección de tecnología que compense las necesidades médicas y apoyando las políticas en salud en todos los niveles ${ }^{[12]}$. La aplicación de las ETS puede ser diversa y empleada en cualquier tecnología sanitaria como: productos farmacéuticos, dispositivos y equipos médicos, procedimientos y sistemas organizativos en atención en salud. No obstante, cada una de estas categorías posee diferencias en el momento de realizar una evaluación de tecnología [3]. Asimismo, estudia las implicaciones médicas, sociales, éticas y económicas del desarrollo, difusión relacionadas con el uso de las tecnologías sanitarias de una manera sistemática, transparente e imparcial, cuyo objetivo es informar para la toma de decisiones considerando aspectos importantes de seguridad, efectividad y las políticas de salud centradas en el paciente, para así lograr la mejor prestación de los servicios de salud ${ }^{[13]}$.

Como se ha mencionado, las ETS pueden ser aplicadas a los dispositivos médicos y no se desarrolla con el ideal de mejorar la relación costo-efectividad, sino a favor de mejorar las decisiones sobre adquisición y/o incorporación para obtener un mayor beneficio clínico en cuanto a seguridad del paciente, eficacia, comodidad del médico, disminución de las terapias y/o hacerlas más agradables para los pacientes. Seguido de promover la eficiencia al proporcionar recomendaciones sobre la adopción de nuevas tecnologías ${ }^{[14]}$. Las ETS se han desarrollado considerablemente en las últimas 3 décadas alrededor del mundo y en Latinoamérica y el Caribe (LAC) en los últimos 15 años se han venido concentrando esfuerzos para su implementación ${ }^{[15]}$.

\section{ETS una revisión global}

A nivel mundial, se han conformado diversas organizaciones que contribuyen y fomentan la cultura de las evaluaciones de tecnología, para mejorar el acceso de los dispositivos médicos controlar las incorporaciones en instituciones de salud, realizando recomendaciones adecuadas a hospitales y clínicas. En países como: España, Inglaterra, Francia, entre otros, se han especializado en la producción técnico-científica de ETS. Dichas evaluaciones, están diseñadas específicamente por grupos interdisciplinarios para su análisis y construcción, enfocándose primordialmente en aspectos de eficacia, seguridad, técnicos y la relación costo-efectividad, para brindar asesoría a los hospitales acerca de los impactos positivos y negativos que tiene la adquisición de determinadas tecnologías médicas. Por tal motivo se realiza una descripción de las diversas agencias que se encuentran constituidas a nivel mundial. 
Europa ha creado regulaciones para diversas tecnologías sanitarias, entre ellas para los dispositivos médicos. Estas normas determinan aspectos sobre el riesgo y funcionalidad para los dispositivos que desean ingresar al mercado ${ }^{[16]}$. Los países europeos han creado procesos consecuentes para la toma de decisiones para lo cual las ETS son aplicadas, pero existe diversidad relacionada con el nivel de desarrollo de los métodos y los procesos de las ETS ${ }^{[17]}$. Por ejemplo, en el caso de los dispositivos médicos es importante considerar el riesgo del dispositivo en relación con la seguridad del paciente; la eficacia y efectividad clínica también son criterios esenciales para producir ETS sólidas. La evidencia científica que se encuentra disponible para los dispositivos médicos no siempre es de alta calidad ${ }^{[18]}$. En ocasiones estos dispositivos o equipos médicos son aplicados para el cuidado del paciente con poco o ninguna evaluación de eficacia o seguridad después de su ingreso al mercado ${ }^{[16]}$. El ideal es contribuir a la transferencia de información entre las diversas organizaciones encargadas de ETS. Actualmente, se discute la armonización de las agencias europeas de evaluación, donde proponen la realización de informes estandarizados en toda la unión europea. Esto proporcionaría, que la creación de informes de evaluación sea menos compleja y más eficiente para todos los países y hospitales, teniendo en cuenta la diversidad de los sistemas de salud y las diferencias culturales ${ }^{[19]}$.

\section{En Canadá, la AHFMR (Alberta Heritage Foundation} for Medical Research) en el año 2006 desarrolló una guía de evaluación de tecnologías en salud, en la cual se identificaron los pasos a seguir para un procedimiento de evaluación de tecnologías en salud. La guía permite calificar los criterios de seguridad, efectividad, eficiencia y conveniencia de la tecnología. También se evidencia criterios asociados a esta guía como el impacto de operación de la tecnología y evaluación financiera. Estos criterios se evalúan con el ideal de escoger la tecnología apropiada para suplir las necesidades de un hospital ${ }^{[20]}$.
En la década de los años 90, se inició una transformación del sector salud en América Latina, la Organización Panamericana de la salud ha trabajado de forma activa en la promoción de la implementación de la ETS, contribuyendo a la mejora y ejecución de los procesos de evaluación, así como el establecimiento de prioridades a el momento de la selección de tecnologías. Se han realizado diversos procesos con diferentes países latinoamericanos, los cuales proporcionaron información acerca del estado actual de la implementación de las ETS, con base al desarrollo de las capacidades humanas, financiamiento, estructura organizativa, procesos, alcance, criterios de decisión, estandarización de la metodología, disposición del uso de datos locales y ayuda internacional en ETS; identificando objetivos a largo plazo y teniendo en cuenta los puntos comunes a nivel regional. Dado lo anterior, se observó que los recursos humanos son insuficientes, además la inversión pública es limitada para la aplicación de las ETS. Los procesos de evaluación no solo se deben limitar a las decisiones políticas de las nuevas tecnologías y se debe realizar con calidad y transparencia ${ }^{[21]}$. Los desarrollos de ETS en Latinoamérica han sido sometidos a un empeño constante por mejorar y hacer más eficiente la asignación de recursos, permitiendo la comparación de estas regiones, con regiones como Europa, donde se han desarrollados panoramas equivalentes ${ }^{[22]}$.

Colombia, en el año 2012 comienza con la estandarización de procesos en el ámbito hospitalario con la creación del IETS y una guía de la evaluación para la adquisición de equipos biomédicos, ayudando al desarrollo del país y proceso de gestión de la tecnología en hospitales como una actividad estandarizada y transparente ${ }^{[23]}$. El objetivo principal del IETS es promover el acceso equitativo, eficiente y sostenible de tecnologías de calidad a todos los ciudadanos. El instituto enfoca las evaluaciones de tecnologías en salud en la evidencia científica o medicina basada en la evidencia, produciendo guías y protocolos sobre medicamentos, dispositivos, procedimientos y tratamientos con el fin 
de recomendar a las autoridades competentes sobre las tecnologías que deben ser cubiertas con recursos públicos a través del Sistema General de Seguridad Social en Salud ${ }^{[24]}$. Se han analizado diversos procesos de adquisición tecnológica en instituciones prestadoras de servicios de salud (IPS) colombianas, con el ideal de identificar las principales formas de adquisición de tecnología biomédica, la forma de identificación y priorización de las necesidades tecnológicas, métodos empleados para la evaluación de dispositivos y equipos médicos, requisitos legales y técnicos exigidos en el momento de la compra, así como la búsqueda de alertas sanitarias nacionales e internacionales en bases de datos relacionadas con los dispositivos. Esto ha llevado a determinar que los manuales metodológicos existentes no contienen los requisitos suficientes que deben ser considerados a nivel hospitalario para la realización de ETS a dispositivos o equipos médicos que van a ser adquiridos o ya están en funcionamiento ${ }^{[13]}$.

\section{ETES para hospitales}

Los dispositivos médicos se emplean habitualmente en todos los procesos de prestación de los servicios de salud; el grado y la complejidad de los problemas que puedan surgir de su uso son muy diversos. La obligación de un programa integral para la gestión de tecnología médica debe garantizar que todas las tecnologías en uso se desempeñen según lo previsto por el fabricante y que sean seguras para los pacientes y el personal que las emplea. A medida que la tecnología hospitalaria continúa evolucionando, el impacto sobre los resultados en el paciente, la operación del hospital y recursos financieros también lo hacen. La gestión de la tecnología en un ambiente hospitalario inicia con una identificación de las necesidades y las capacidades, teniendo en cuenta el crecimiento acelerado de nuevas tecnologías para la mejora de la prestación de los servicios de salud ${ }^{[25]}$. Las evaluaciones de tecnología en salud para hospitales son actividades que informan acerca de las decisiones de gestión relacionadas con diversos tipos de tecnologías de salud. Los hospitales son principalmente el punto de entrada para las nuevas tecnologías y estas nuevas tecnologías pueden reemplazar o agregar a las tecnologías existentes. Por lo tanto, los procesos y métodos de organización y realización de ETS deben tener un enfoque multidisciplinario, sistemático y basado en la evidencia.

Los métodos y la evidencia científica utilizada en hospitales basados en ETS (HB-ETS) son generalmente los mismos que utilizan para emprender ETS a nivel nacional o regional. No obstante, la evidencia preliminar de los hospitales muestra que las herramientas de evaluación y la información requerida para la toma de decisiones a nivel hospitalario difieren de las utilizadas a nivel nacional o regional. De esta manera, las HB-ETS tienen como objetivo ser de utilidad para los responsables de la toma de decisiones del hospital, es crucial conocer sus necesidades informativas ${ }^{[26]}$.

A nivel hospitalario, Europa también ha dedicado tiempo para el desarrollo de ETS a nivel hospitalario. Los gerentes o administradores de diversos hospitales europeos demuestran la necesidad de información para la toma de decisiones sobre nuevos tratamientos, para esto han adoptado modelos conocidos y aceptados para la producción de ETS como lo es el modelo básico de EUnetHTA. Los hospitales consideran los criterios clínicos, económicos, seguridad y organizativos como información relevante para nuevos tratamientos. Los criterios para las ETS basadas en hospitales deben reflejar la necesidad informativa a los gerentes o administradores de las instituciones de salud para decidir si invertir o no en nuevas tecnologías o tratamientos ${ }^{[27]}$.

Instituciones nacionales independientes a nivel europeo proporcionan información relevante de criterios de evaluación implementados a nivel hospitalario. Una de ellas es la Red europea para la evaluación de tecnologías sanitarias - EUnetHTA, esta es una de las agencias europeas más representativas a nivel mundial, la 
cual propone uno de sus más utilizados modelos, el Core Model desarrollado principalmente por agencias nacionales de ETS, el cual incluye diversos criterios de evaluación que son necesarios para la toma de decisiones para la adquisición de nuevas tecnologías. Aunque la EUnetHTA es una institución nacional muchos hospitales adecuan los dominios de evaluación que esta les ofrece. Sin embargo, no se conoce completamente el grado en que los dominios del Core Model cubre las necesidades de información que necesitan los tomadores de decisión en el hospital ${ }^{[28]}$. Los criterios que establece EUnetHTA (entendidos como "dominios del modelo básico de EUnetHTA") son los siguientes:

1. Problemas de salud y uso de la tecnología

2. Descripción y características de la tecnología

3. Seguridad

4. Eficacia clínica

5. Costos y evaluación económica

6. Análisis ético

7. Aspectos organizacionales

8. Pacientes y aspectos sociales

9. Aspectos legales

Con el pasar de los años, ha crecido la necesidad de transferir la metodología de las ETS para contribuir en la toma de decisiones gerenciales a nivel hospitalario. La adopción de la evaluación de tecnología de salud basada en hospitales (AdHopHTA) es una de las instituciones nacionales que está estructurada específicamente para la aplicación de ETS en hospitales. Teniendo en cuenta que los hospitales funcionan con limitaciones en los recursos, se ha difundido la cultura de la medicina basada en la evidencia para que sea implementada por los encargados de formular políticas, por los administradores y gerentes de hospitales, por los médicos y por otros profesionales, con el ideal de aportar la suficiente información verídica para el desarrollo de la práctica clínica y los procesos institucionales con el objetivo de identificar herramientas que faciliten la toma de decisiones yofrezacn metodologías técnicas que respalden decisiones afines con asuntos fundamentales, ya sea la adquisición o la asignación de recursos tecnológicos ${ }^{[29]}$.

\section{Medicina basada en la evidencia}

La medicina basada en la evidencia (MBE), es una de las prácticas más utilizadas hoy en día en el ámbito médico para fortalecer la toma de decisiones por medio de la implementación de pruebas científicas provenientes de estudios de investigación. A continuación, se describirá la fuerza que ha cobrado este método y las aplicaciones que ha tenido en las evaluaciones de tecnología en salud. La MBE es la combinación de la práctica clínica con la evidencia de la investigación actual, en conjunto con las preferencias de los

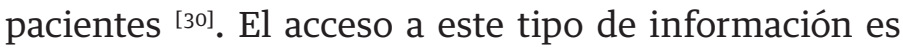
limitado, la incapacidad de las fuentes tradicionales para satisfacer esta necesidad y la escasez de información actualizada se ha convertido en una dificultad. La MBE ha sido planteada como una solución a estos problemas y a los nuevos retos que se presentan en la atención de la medicina ${ }^{[31]}$. Las entidades encargadas de ETS deben revisar y validar los datos de las industrias de equipos y dispositivos médicos, con base al estudio de literatura de evidencia científica en los diversos criterios de evaluación y no en la información que proporciona el fabricante ${ }^{[32]}$. Sin embargo, como se ha mencionado con anterioridad, encontrar publicaciones disponibles de este tipo de información no es fácil. Por tal motivo, es necesario formular una adecuada pregunta de investigación que permita acceder a información de gran importancia ${ }^{[33]}$.

La evidencia tiene diversas fases y clasificaciones. Cuando se habla de la base de la evidencia se hace referencia, a la información empleada para realizar informes de evaluación; es decir, se toma como base la información que otorga el fabricante, datos obtenidos durante una búsqueda sistemática de bibliografía o ambas. El tipo de evidencia puede distinguir pruebas directas, como: ensayos directos o ensayos clínicos 
aleatorios ECA bien estructurados, revisiones sistemáticas de literatura (RSL) o metaanálisis. Si la información es insuficiente se pueden tomar otro tipo de estudios, de acuerdo con la clasificación de la evidencia para apreciar los efectos que se obtienen en los diversos tratamientos. Es sustancial obtener decisiones clínicas que estén fundamentadas en los conceptos científicos bien consolidados. La MBE platea tomar como base la jerarquización de la pirámide de la evidencia y así tener un mejor acceso a información confiable, teniendo en cuenta diseños aptos en la investigación clínica, como Las RSL y los meta-análisis se encuentran establecidos como estudios de mayor confiabilidad, seguido por los ensayos clínicos controlados aleatorios, en el último nivel encuentran los consensos de expertos que pueden ser empleados cuando no existe ninguna evidencia científica ${ }^{[34]}$.

\section{Criterios de evaluación basados en evidencia}

\section{Seguridad del paciente}

Cuando se hace referencia a seguridad, según la OMS es: "La reducción del riesgo de daños innecesarios hasta un mínimo aceptable..." [35]. Según otros autores en cuanto a seguridad del paciente la definen como: “evitar, prevenir o amortiguar los resultados adversos o el daño derivado del proceso de la asistencia sanitaria” [35]. Seguidamente la cultura de seguridad es la unión de valores y normas comunes a los individuos dentro de una misma organización e involucra un modelo mental simultáneo que posiciona la seguridad como un objetivo a seguir [35]. Wang et al., 2017 argumenta que numerosos accidentes acontecen cada día en muchas organizaciones de todo el mundo, que implican lesiones a las personas, contaminación ambiental y daños consecuentes a la reputación, por lo tanto, la gestión de seguridad es un elemento crucial para realizar una gestión efectiva organizacional. Es significativo tomar una decisión efectiva de seguridad fundada en información confiable y suficiente. No obstante, diversas fallas en la gestión de seguridad se deben a la falta de información para la toma de decisiones, teniendo en cuenta que los hechos son la base fundamental para la toma de decisiones, es necesario centrar los esfuerzos para buscar evidencia relevante para el manejo de gestión de la seguridad y dar soluciones efectivas a los problemas en particular con seguridad ${ }^{[36]}$. La seguridad del paciente es uno de los puntos clave en los sistemas de salud y uno de los más grandes problemas en salud pública, debido al incremento de eventos adversos por usos inadecuados en las tecnologías médicas, desencadenando terribles desenlaces y significativas implicaciones financieras. Por tal motivo, se ha incentivado a las instituciones a contemplar casos de alertas sanitarias para la gestión de seguridad en ambientes poco seguros, con el fin de brindar las mejores garantías para los pacientes y disminuir la incidencia de eventos adversos. En el área de la salud, recientemente se han interesado en la evaluación de reportes de casos de seguridad, en especial en dispositivos médicos para mejorar las técnicas de gestión de la seguridad de forma dinámica y rigurosa. Para la atención médica la implementación de reportes de estos casos es una práctica poco empleada y que posee insuficiente evidencia. Sin embargo, los reportes de seguridad pueden generar una mejor perspectiva de exposición al riesgo en lugar de políticas reguladoras que demuestren los niveles de aceptación de la seguridad [37].

\section{Eficacia Clínica basada en la evidencia}

Los estudios de eficacia son creados para proporcionar efectos que apoyan a los médicos a seleccionar entre varias opciones de tratamiento, por medio de pruebas que se realizan a las hipótesis de investigación; es decir, el beneficio en particular que causa una determinada intervención, realizada en condiciones ideales ${ }^{[38]}$. Por lo general, los datos sobre los efectos o desenlaces de una intervención se seleccionan bajo el argumento de los ensayos clínicos aleatorios (ECA). La descripción de este estudio es ideal para demostrar la eficacia de determinada intervención debido a los 
datos que proporciona y que contribuye a la disminución de problemas de confusión, sesgo de información y sesgo de selección. En efecto, es un desafío inferir los resultados de los ECA de las intervenciones en la práctica clínica, a causa de las limitaciones relacionadas con la implementación y disponibilidad de evidencia de eficacia ${ }^{[39]}$. Los ECA son la una metodología sólida y efectiva para demostrar de forma científica el análisis de la eficacia de una intervención clínica ${ }^{[40]}$.

\section{Economía basada en la evidencia}

La evaluación económica se ocupa del análisis sistemático de los costos y beneficios originados por la comparación minuciosa y precisa de tratamientos y/o dispositivos médicos alternativos para comprobar cuáles ofrecen mejor bienestar a pacientes específicos y a la sociedad. Estas evaluaciones unen la medicina basada en la evidencia a las pretensiones de la sociedad y usuarios en especial. El objetivo es mejorar la eficiencia en la distribución de los recursos y atención de la salud, así como lograr equidad social, no la limitación en los gastos de atención médica. La producción técnica de las evaluaciones es apta para científicos clínicos y economistas, encargados de la construcción de información para adopción y gastos en salud. Por otro lado, las evaluaciones económicas tienden a un error común: se centran únicamente en determinar el costo o la diferencia de costo entre dos tecnologías médicas. Sin embargo, el ideal es combinar información de costos y beneficios para apoyar a los tomadores de decisiones en su evaluación ${ }^{[41]}$. Las evaluaciones económicas y los análisis de costos son fundamentales para notificar la determinación de prioridades y las decisiones de distribución de recursos en salud. Estos análisis pueden tener diversos enfoques, como: costo-efectividad, costo-utilidad, costo-beneficio-minimización de costo, impacto presupuestal. Es importante conocer la perspectiva para la cual se realiza el análisis; es decir, el enfoque social es mucho más completo y considera todos los costos y consecuencias asociadas a la intervención o enfermedad. Otras pers- pectivas como los proveedores, el sistema de atención médica o los profesionales, utilizan otros criterios económicos al evaluar ${ }^{[42]}$. Finalmente, los sistemas de salud tienen el objetivo de proporcionar servicios de alta calidad a la sociedad, permitiendo una rápida acogida a la innovación que mejore el alcance para producir servicios de salud en cantidad para satisfacer las necesidades de la comunidad, haciéndolo de manera eficiente y disminuyendo el gasto innecesario ${ }^{[41]}$.

\section{DISCUSIÓN}

En este artículo se presentó una revisión del estado del arte acerca de las evaluaciones de tecnologías efectuadas en diversos países, identificando cuál es el enfoque dado a nivel hospitalario y cuál es el objetivo para el que están destinadas. La instauración de diversas organizaciones especializadas en ETS se está convirtiendo en un elemento fundamental para identificar las tecnologías emergentes, con el ideal de detectarlas oportunamente. Estas organizaciones se han comprometido fuertemente para contribuir con el desarrollo de las ETS.

La gestión tecnológica en salud es uno de los procesos fundamentales desarrollados en las instituciones de salud. De este proceso se desencadena la evaluación de tecnología a nivel hospitalario contribuyendo a la toma de decisiones realizadas por gerentes y administradores de las instituciones de salud y así obtener óptimos resultados para una buena implementación de los recursos financieros.

Las ETS se definen como el procesos científico, multidisciplinario y multidimensional que permite el análisis comparativo entre dos tecnologías concentrando los aspectos positivos y negativos, generando alternativas, proporcionando la mejor selección de acuerdo con las necesidades clínicas para la toma de decisiones ${ }^{[12]}$. Las ETS pueden aplicarse en diversas tecnologías como los fármacos, dispositivos médicos, procedimientos entre otros. 
El desarrollo que se ha evidenciado a nivel mundial ha proporcionado efectos positivos para la incorporación de nuevas tecnologías en los países y, posteriormente, a las instituciones de salud.

Los países europeos tienen el ideal de transferir información entre las diversas organizaciones encargadas del desarrollo de ETS ${ }^{[19] . ~ E n ~ L a t i n o a m e ́ r i c a, ~}$ proporcionan medidas que ayudan a la mejora de los procesos de evaluación enfocados en el desarrollo de diversos aspectos, como las capacidades humanas, la financiación o las estructuras organizativas, entre otras. En Colombia, los manuales metodológicos para las evaluaciones de tecnologías existentes no dan cobertura a las necesidades hospitalarias para la implementación de ETS.

La aplicación de las ETS a nivel hospitalario es una gran herramienta multidisciplinaria, sistémica y basada en evidencia, que ayudan a determinar las decisiones de la gestión realizada con las diversas tecnologías sanitarias que se desean adquirir. Es importante considerar que los hospitales y los pacientes son los principales usuarios de las nuevas tecnologías que pueden reemplazar o agregar a las ya existentes.
Las ETS evalúan diversos criterios, como la seguridad y la eficacia económico-social, los cuales son fundamentales al realizar una evaluación de tecnología. Estos dominios deben ser evaluados con medicina basada en evidencia, la cual suministra información verídica para los procesos de evaluación. Aunque este tipo de información es escasa y de baja o mediana calidad, se debe de buscar información de alta calidad, debido que es importante enfocar las ETS con este tipo de datos.

\section{CONCLUSIONES}

Las evaluaciones de tecnología son procesos fundamentales que proporcionan información relevante para la toma de decisiones para las incorporaciones de nuevos dispositivos médicos.

La implementación de las ETS a nivel hospitalario contribuye a mejorar la seguridad de los pacientes por medio de la implementación de tecnología eficaz y económicamente rentable para las instituciones de salud.

La toma de decisiones con base a evidencia científica permite realizar procesos trasparentes, acertados y justificados para una mejor regulación y administración de los recursos. 


\section{REFERENCIAS}

[1] Tejada MC, Ibañez CGR. Technical and clinical evaluation of biomedical technology in the process of acquisition: a focus in healthcare technology. Rev Ing Biomédica [Internet]. 2008;2(4):3445. Available from: http://repository.eia.edu.co/revistas/index.php/ BME/article/view/52

[2] OMS. Formulación de políticas sobre dispositivos médicos Serie de documentos técnicos de la OMS sobre dispositivos médicos. 2012;

[3] Migliore A. Technology assessment of innovative medical devices in Europe. Expert Rev Med Devices [Internet]. 2016;13(3):217-9. Available from: http://www.tandfonline.com/doi/full/10.1586/1743 4440.2016.1146588

[4] Oortwijn W, Broos P, Vondeling H, Banta D, Todorova L. Mapping of Health Technology Assessment in Selected Countries. Int J Technol Assess Health Care. 2013;29(4):424-34.

[5] Kuchenbecker R, Polanczyk CA. Institutionalizing Health Technology Assessment in Brazil : Challenges Ahead. Value Heal Reg Issues [Internet]. 2012;1(2):257-61. Available from: http://dx. doi.org/10.1016/i.vhri.2012.09.009

[6] Ciani O, Blankart CR, Hatz M, Taylor RS. Health Technology Assessment of Medical Devices : A Survey of Non-European Union Agencies. 2015;3(305694):154-65.

[7] Barrientos Gómez JG, Marín Castro AE, Becerra Ruiz L, Tobón Arango MA. La evaluación de nuevas tecnologías en salud en hospitales: revisión narrativa. Med UPB [Internet]. 2016;35(2):12034. Available from: https://revistas.upb.edu.co/index.php/Medicina/ article/view/7146

[8] Vilcahuamán L, Rivas R. Healthcare Technology Planning and Acquisition. Healthc Technol Manag Syst [Internet]. 2017;47-70. Available from: $h t t p: / / l i n k i n g h u b . e l s e v i e r . c o m / r e t r i e v e / p i i /$ B9780128114315000047

[9] OMS. Evaluación de tecnologías sanitarias aplicada a los dispositivos médicos. Ser Doc Tec la OMS sobre Dispos médicos. 2012;1-44.

[10] Restrepo G. El Concepto y Alcance de la Gestión Tecnológica. 2001.

[11] OMS. Global atlas of medical devices [Internet]. WHO Medical

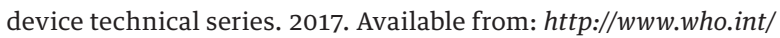
medical_devices

[12] Pecchia L, Castaldo R, Melillo P, Bracale U, Craven M, Bracale M. Early Stage Healthcare Technology Assessment. Clin Eng. 2016;95115.

[13] Salazar-Flórez KJ, Botero-Botero S, Jiménez-Hernández CN. Adquisición de tecnología biomédica en IPS colombianas: Comparación y mejores prácticas. Rev Gerenc y Polit Salud. 2016;15(31):88-118.

[14] Jaffray DA. World congress on medical physics and biomedical engineering, june 7-12, 2015, Toronto, Canada. IFMBE Proc. 2015;51(October).

[15] Pichon-Riviere A, Soto NC, Augustovski FA, Martí SG, SampietroColom L. Health technology assessment for decision making in Latin America: Good practice principles. Int J Technol Assess Health Care. 2018;34(3):241-7.
[16] Fuchs S, Olberg B, et al. Testing a new taxonomic model for the assessment of medical devices: Is it plausible and applicable? Insights from HTA reports and interviews with HTA institutions in Europe. Health Policy (New York) [Internet]. 2018; Available from: https://doi.org/10.1016/j.healthpol.2018.03.004

[17] García-Mochón L, Espín Balbino J, Olry de Labry Lima A, Caro Martinez A, Martin Ruiz E, Pérez Velasco R. HTA and decisionmaking processes in Central, Eastern and South Eastern Europe: Results from a survey. Health Policy (New York) [Internet]. 2016; Available from: http://dx.doi.org/10.1016/j.healthpol.2017.03.010

[18] Fuchs S, Olberg B, et al. HTA of medical devices: Challenges and ideas for the future from a European perspective. Health Policy (New York) [Internet]. 2017;121(3):215-29. Available from: http://dx.doi.org/10.1016/j.healthpol.2016.08010

[19] Rüther A. HTA und aktuelle Herausforderungen : Harmonisierung, Real World Data und Surrogatparameter HTA - How to tackle pressing challenges : International Harmonization, Real World Data , and Surrogates HTA-Netzwerk : Zusammenarbeit. 2018;14(1):1-11.

[20] Usaquen SP, Cano A, Troncoso DM, Bonilla N, Almeida RT De. El uso de la Evaluación de Tecnologías en Salud para la Adquisición de Tecnología en Hospitales. 2017;27-34.

[21] Rosselli D, Quirland Lazo C, Csanádi M, Ruiz de Castilla EM, González NC, Valdés J, et al. HTA Implementation in Latin American Countries: Comparison of Current and Preferred Status. Value Heal Reg Issues [Internet]. 2017;14:20-7. Available from: http://dx.doi.org/10.1016/j.vhri.2017.02.004

[22] Skaltsa K, Allen N, Van Engen A, Blogg K. An archetype for classification and comparison of HTA activities in Latin America. Value Heal [Internet]. 2014;17(7):A443. Available from: https://doi.org/10.1016/j.jval.2014.08.1170

[23] González D. Herramienta de evaluación para la adquisición de equipos biomédicos. 2014;97. Available from: http://hdl.handle.net/11190/300

[24] Instituto de Evaluación Tecnológica en Salud (IETS) Colombia. Acta de Constitución y Estatuto IETS. 2012;1-18.

[25] Pallikarakis N, Bliznakov Z. Hospital Technology Management. Clin Eng [Internet]. 2016;47-61. Available from: http://linkinghub. elsevier.com/retrieve/pii/B9780128037676000052

[26] Partners A project. THE AdHopHTA HANDBOOK. 2015; Available from: $h t t p: / / w w w . a d h o p h t a . e u$

[27] Kidholm K, Ølholm AM, Birk-Olsen M, Cicchetti A, Fure B, Halmesmäki E, et al. Hospital managers' need for information in decision-making - An interview study in nine European countries. Health Policy (New York) [Internet]. 2015;119(11):1424-32. Available from: http://dx.doi.org/10.1016/j.healthpol.2015.08.011

[28] Ja TE, Union E, Programme H. EUnetHTA JA2 WP8 DELIVERABLE HTA Core Model ${ }^{\circledR}$ Online User guide. 2015;2:1-27. Available from: www.eunethta.eu

[29] Cicchetti A, Marchetti M, Dibidino R, Corio M. Hospital Based Health Technology Assessment World-Wide Survey. Hosp Based Heal Technol Assess Sub-Interest Gr. 2008;41. 
[30] Abdulwadud O, Azazh A, Mekasha A, Bacha T, Nigatu B, Debebe F, et al. African Journal of Emergency Medicine Cochrane, evidencebased medicine and associated factors : A cross-sectional study of the experiences and knowledge of Ethiopian specialists in training. African J Emerg Med [Internet]. 2019;(November 2018):0-1. Available from: https://doi.org/10.1016/j.afjem.2019.01.005

[31] Kalavani A, Kazerani M, Shekofteh M. Acceptance of evidence based medicine (EBM) databases by Iranian medical residents using unified theory of acceptance and use of technology (UTAUT). Heal Policy Technol [Internet]. 2018;7(3):287-92. Available from: https://doi.org/10.1016/j.hlpt.2018.06.005

[32] García-Mochón L, Espín Balbino J, et al. HTA and decision-making processes in Central, Eastern and South Eastern Europe: Results from a survey. Health Policy (New York) [Internet]. 2019;123(2):182-90. Available from: http://dx.doi.org/10.1016/j.healthpol.2017.03.010

[33] Gavín Benavent P. Ciencias De La Salud Basadas En La Evidencia. Concepto Y Antecedentes. Productos Basados En La Evidencia. Ciencias la Salud Basadas en la Evid [Internet]. 2011;1-14. Available from: http://www.ics-aragon.com/cursos/salud-publica/2014/pdf/ M4T04.pdf

[34] Rosner AL, Ph D, Hon LLD. Evidence-based medicine : Revisiting the pyramid of priorities. J Bodyw Mov Ther [Internet]. 2012;16(1):42-9. Available from: http://dx.doi.org/10.1016/i.jbmt.2011.05.003

[35] Rocco C, Garrido A. Seguridad Del Paciente Y Cultura De Seguridad. Rev Médica Clínica Las Condes [Internet]. 2017;28(5):785-95. Available from: http://linkinghub.elsevier.com/retrieve/pii/ So716864017301268

[36] Wang B, Wu C, Shi B, Huang L. Evidence-based safety (EBS) management: A new approach to teaching the practice of safety management (SM). J Safety Res [Internet]. 2017;63:21-8. Available from: https://doi.org/10.1016/i.jsr.2017.08.012
[37] Sujan MA, Habli I, Kelly TP, Pozzi S, Johnson CW. Should healthcare providers do safety cases ? Lessons from a crossindustry review of safety case practices. Saf Sci [Internet]. 2016;84:181-9. Available from: http://dx.doi.org/10.1016/j.ssci.2015.12.021

[38] Minneci PC, Deans KJ. Seminars in Pediatric Surgery Clinical trials. Semin Pediatr Surg [Internet]. 2018;27(6):332-7. Available from: https://doi.org/10.1053/j.sempedsurg.2018.10.003

[39] Makady A, Ham R, Boer A De, Hillege H, Klungel O, Goettsch W. Policies for Use of Real-World Data in Health Technology Assessment ( HTA ): A Comparative Study of Six HTA Agencies. Value Heal [Internet]. 2017;20(4):520-32. Available from: http://dx.doi.org/10.1016/j.jval.2016.12.003

[40] Pokorny MR, De Rooij M, Duncan E, Schröder FH, Parkinson R, Barentsz JO, et al. Prospective study of diagnostic accuracy comparing prostate cancer detection by transrectal ultrasoundguided biopsy versus magnetic resonance (MR) imaging with subsequent mr-guided biopsy in men without previous prostate biopsies. Eur Urol. 2014;66(1):22-9.

[41] Fragoulakis, V., Mitropoulou, C., Williams, M. S., \& Patrinos GP. Economic Evaluation in Health Care : Evidence-Based Medicine and Evidence-Based Health Economics. Econ Eval Heal Care Econ Eval Genomic Med. 2015;1-8.

[42] Lichterfeld-Kottner A, Hahnel E, Blume-Peytavi U, Kottner J. Systematic mapping review about costs and economic evaluations of skin conditions and diseases in the aged. J Tissue Viability [Internet]. 2017;26(1):6-19. Available from: http://dx.doi.org/10.1016/j.jtv.2016.07.002 